Pacific Journal of Mathematics

FIXED POINT SET OF PRODUCTS AND CONES 


\title{
FIXED POINT SETS OF PRODUCTS AND CONES
}

\author{
John R. Martin, Lex G. Oversteegen \\ AND E. D. TYMCHATYN
}

\begin{abstract}
A space $X$ is said to have the complete invariance property (CIP) if every nonempty closed subset of $X$ is the fixed point set of some self-map of $X$. Examples are given to show that for the class of locally connected continua, the operations of taking products, cones, and strong deformation retractions need not preserve CIP. In fact, it is shown that the operations of taking products and cones do not preserve CIP for $L C^{\infty}$ continua.
\end{abstract}

1. Introduction. A subset $K$ of a space $X$ is called a fixed point set of $X$ if there is a continuous function $f: X \rightarrow X$ such that $f(x)=x$ iff $x \in K$. In [8, p. 553] L. E. Ward, Jr. defines a space $X$ to have the complete invariance property (CIP) if every nonempty closed subset of $X$ is a fixed point set of $X$. Examples 4.3, 5.1 and 6.1 in [4] are examples of non-locally connected continua which show that CIP is not preserved by the operations of taking strong deformation retractions, products and cones. The purpose of this paper is to construct locally connected examples showing that CIP is not preserved by the above operations and, in fact, provide examples which answer Questions 5.2, 6.3 and 6.4 in [4]. In particular, we show that if $X$ is a locally connected continuum possessing CIP, then $X \times I$ and Cone $(X)$ need not have CIP. Moreover, it is shown that it is possible for $X$ to be either a 1-dimensional continuum or an $L C^{\infty}$ continuum.

2. Notation and terminology. The terminology used in this paper may be found in [1]. In particular, Hilbert space $E^{\omega}$ with metric $\rho$ and Euclidean $n$-dimensional space $E^{n}$ are as defined in [1, pp. 10-11]. For $k=1,2,3, \cdots$, let $a_{k}$ denote the point in $E^{\omega}$ given by the formula $a_{k}=(1 / k, 0,0,0, \cdots)$, and let $a_{0}$ denote the origin of $E^{\omega}$. Let $S_{k}^{n}$ denote the $n$-dimensional sphere in $E^{\omega}$ consisting of all the points $x=\left(x_{1}, x_{2}, x_{3}, \cdots\right)$ such that $\rho\left(x, a_{k}\right)=1 / k$ and such that $x_{i}=0$ for $i>n+1$. Then we define

$$
A^{n}=\bigcup_{k=1}^{\infty} S_{k}^{n}, A_{m}^{\infty}=\bigcup_{k=m}^{\infty} S_{k}^{k}
$$

A point $p$ in a space $X$ is said to be homotopically stable if for every deformation $H: X \times I \rightarrow X, H(p, t)=p$ for all $t \in I$. For instance, $a_{0}$ is a homotopically stable point in each of the spaces 
$A^{n}$ and $A_{m}^{\infty}$.

3. Products. We first consider some variations of a property considered by L. E. Ward, Jr. in [8, Theorem 1].

Definitions 3.1. (1) A space $X$ has property $Q$ if for every nonempty closed subset $K$ of $X$ there is a point $p \in K$, a retract $R$ of $X$ containing $K$, and a deformation $H: R \times I \rightarrow R$ such that $H(x, t) \neq x$ if $x \neq p$ and $t>0$.

(2) If in (1) we omit $p$ and stipulate that $H(x, t) \neq x$ if $x \notin K$ and $t>0$, then we say that $X$ has property $Q$ (weak).

(3) A space $X$ has property $W$ if for every point $p \in X$ there is a deformation $H: X \times I \rightarrow X$ such that $H(x, t) \neq x$ if $x \neq p$ and $t>0$.

(4) If in (3) $H(x, t) \neq x$ whenever $t>0$, we say that $X$ has property $W$ (strong).

We note that if $X$ is a space having property $W$ (strong) and $Y$ is an arbitrary space, then the product space $X \times Y$ has property $W$ (strong). Since $W$ (strong) $\Rightarrow W \Rightarrow Q \Rightarrow Q$ (weak), the following proposition shows that any metric space satisfying one of these four properties has CIP.

Proposition 3.2. Any metric space $(X, \delta)$ having property $Q$ (weak) has CIP.

Proof. Let $K$ be a nonempty closed subset of $X$, and let $R$ and $H$ be as in Definition (2). We may assume that $\delta \leqq 1$, and let $r: X \rightarrow R$ be a retraction of $X$ onto $R$. Define a map $f: X \rightarrow X$ by

$$
f(x)=H(r(x), \delta(r(x), K)),(x \in X) .
$$

Then it is easy to check that $f$ is a self-map of $X$ whose fixed point set is $K$.

Proposition 3.3. Let $X, Y$ be spaces such that one has property $Q$ and the other has property $W$. Then $X \times Y$ has property $Q$.

Proof. Suppose that $X$ has property $Q$ and $Y$ has property $W$. Let $K$ be a nonempty closed subset of $X \times Y$. Let $\pi$ denote the natural projection of $X \times Y$ onto $X$. Then there is a point $p \in \pi(K)$, a retract $R$ of $X$ containing $\pi(K)$, and a homotopy $F: R \times I \rightarrow R$ such that

$$
\begin{aligned}
& F(x, 0)=x \\
& F(x, t) \neq x \text { if } x \neq p \text { and } t>0 .
\end{aligned}
$$


Let $q$ be a point in $Y$ such that $(p, q) \in K$. Then there is a homotopy $G: Y \times I \rightarrow Y$ such that

$$
\begin{aligned}
& G(y, 0)=y, \\
& G(y, t) \neq y \text { if } y \neq q \text { and } t>0 .
\end{aligned}
$$

Now $(p, q) \in K \subset R \times Y$ and $R \times Y$ is a retract of $X \times Y$. Define a homotopy $H:(R \times Y) \times I \rightarrow R \times Y$ by

$$
H((x, y), t)=(F(x, t), G(y, t)), \quad(x \in R, y \in Y, t \in I) .
$$

Then $H((x, y), 0)=(x, y)$, and $H((x, y), t) \neq(x, y)$ if $(x, y) \neq(p, q)$ and $t>0$. Therefore $X \times Y$ has property $Q$.

As a corollary to Propositions 3.2 and 3.3, we obtain the following theorem.

THEOREM 3.4. Let $X, Y$ be metric spaces such that one has property $Q$ and the other has property $W$. Then $X \times Y$ has CIP.

We remark that the class of spaces satisfying property $W$ includes any space which admits a strongly convex metrization (see $[1$, p. 219] and [8, p. 554]), compact manifolds without boundary [6], and all compact triangulable manifolds with or without boundary [7]. Furthermore, property $W$ (strong) holds for the case where one of the above manifolds has Euler characteristic equal to zero, or for the case where the space is a metric group which contains an arc $[4$, p. 1028].

The following example answers (5.2) in [4] and shows that the hypothesis of property $Q$ in Theorem 3.4 cannot be replaced by property $Q$ (weak).

Example 3.5. For $k=1,2,3, \cdots$, let $p_{k}$ denote the unique point on the upper semicircle of $S_{1}^{1} \subset E^{2}$ whose first coordinate is $1 / k$. Let $B_{1}, B_{2}, B_{3}, \cdots$ be a null sequence of disjoint copies of $A^{1}$ lying in the disk bounded by $S_{1}^{1}$ such that

(1) For each $k=1,2,3, \cdots, B_{k} \cap S_{1}^{1}=\left\{p_{k}\right\}$ where $p_{k}$ is the point in $B_{k}$ which corresponds to the point $a_{0}$ in $A^{1}$. Then,

(2) $\lim _{k \rightarrow \infty} B_{k}=\left\{a_{0}\right\} \subset S_{1}^{1}$.

Define $Y=S_{1}^{1} \cup \bigcup_{k=1}^{\infty} B_{k}$.

Then $Y$ is a 1-dimensional planar Peano continuum. It follows from the proof of Theorem 1 in [5] that $Y$ has property $Q$ (weak) and thus has CIP. However, $Y \times I$ does not have CIP. To see this, let

$$
C=\left\{\left(a_{0}, 0\right)\right\} \cup \bigcup_{k=1}^{\infty} B_{2 k-1} \times\{0\},
$$




$$
D=\left\{\left(a_{0}, 1\right)\right\} \cup \bigcup_{k=1}^{\infty} B_{2 k} \times\{1\} .
$$

Suppose $f: Y \times I \rightarrow Y \times I$ is a mapping whose fixed point set is $C \cup$ $D$. Since the points $a_{0}, p_{1}, p_{2}, p_{3}, \cdots$ are homotopically stable points of $Y$, it follows that $f(\{q\} \times I) \subset\{q\} \times I$ for any point $q \in\left\{a_{0}, p_{1}, p_{2}\right.$, $\left.p_{3}, \cdots\right\}$. Consequently, if $0<t<1$ and $f\left(p_{k}, t\right)=\left(p_{k}, s\right)$, it follows that $s<t$ if $k$ is odd, and $s>t$ if $k$ is even. Therefore $f\left(a_{0}, t\right)=$ $\left(a_{0}, t\right)$ for all $t \in I$ which is a contradiction.

The following examples are higher dimensional analogues of Example 3.5.

EXAMPLE 3.6. If we use the same notation as was used in Example 3.5 and let $B_{k}$ be homeomorphic to $A^{n}\left(A_{k}^{\infty}\right)$ for $k=1,2$, $3, \cdots$, then we obtain an $L C^{n}\left(L C^{\infty}\right)$ continuum $Y^{n}\left(Y^{\infty}\right)$ such that $Y^{n} \times I\left(Y^{\infty} \times I\right)$ does not have CIP. Moreover, it is easy to show that $Y^{n}\left(Y^{\infty}\right)$ satisfies property $Q$ (weak) and hence has CIP.

To see this for $Y^{\infty}$, suppose $C$ is a nonempty closed subset of $A_{1}^{\infty}=\bigcup_{k=1}^{\infty} S_{k}^{k}$. Let $R$ denote the retract of $A_{1}^{\infty}$ defined by

$$
R=S_{1}^{1} \cup \cup\left\{S_{k}^{k} \mid C \cap\left(S_{k}^{k}-\left\{a_{0}\right\}\right) \neq \varnothing\right\} .
$$

We first construct a deformation of $R$ which shows that $A_{1}^{\infty}$ has property $Q$ (weak) by constructing deformations on each of the $S_{k}^{k}$ lying in $R$. For each $k>1$ such that $S_{k}^{k} \subset R$, choose one point $c_{k} \in$ $C \cap\left(S_{k}^{k}-\left\{a_{0}\right\}\right)$. If $a_{0} \in C$, consider a deformation of $S_{k}^{k}$ which fixes $c_{k}$ and $a_{0}$ for all values of $t$, and for $t>0$ moves points along radial rays from the point $c_{k}$ to the point $a_{0}$ at infinity. To obtain the required deformation of $R$ we use the above deformations together with a deformation of $S_{1}^{1}$ whose terminal map is a translation of $S_{1}^{1}$ which fixes the point $a_{0}$ at infinity. If $a_{0} \notin C$, then there are only finitely many values for $k$ such that $S_{k}^{k} \subset R$. In this case, we consider a deformation of $S_{1}^{1}$ whose terminal map is a rotation of $S_{1}^{1}$, and we adjust the previous arguments so as to obtain deformations of the $S_{k}^{k}$ into $R$ which agree with the new deformation of $S_{1}^{1}$. This shows that $A_{1}^{\infty}$ has property $Q$ (weak) and these arguments can be modified to show that $Y^{\infty}$ has property $Q$ (weak).

4. Cones. The following example answers Questions 6.3 and 6.4 in [4] by showing that the cone over a 1-dimensional Peano continuum having CIP need not have CIP.

EXAMPLE 4.1. First we construct a 1-dimensional planar Peano continuum $X$ such that every point in $X$ is homotopically stable. We start the construction by considering the Hawaiian earring $A^{1}=$ 
$\bigcup_{k=1}^{\infty} S_{k}^{1}$. Let $B_{1}^{n}, B_{2}^{n}, B_{3}^{n}, \cdots$ be a null sequence of disjoint copies of $A^{1}$ lying in the disk bounded by $S_{n}^{1}$ and lying in the exterior of the disk bounded by $S_{n+1}^{1}$ such that

(1) For each $k=1,2,3, \cdots, B_{k}^{n} \cap S_{n}^{1}=\left\{b_{k}^{n}\right\}$ where $b_{k}^{n}$ denotes the unique homotopically stable point of $B_{k}^{n}$.

(2) The set $\left\{b_{k}^{n} \mid k=1,2,3, \cdots\right\}$ is a dense subset of $S_{n}^{1}$.

The first stage of our construction yields the space $A^{1} \cup \bigcup_{k, n=1}^{\infty} B_{k}^{n}$. In the second stage, the above process is repeated for each of the Hawaiian earrings $B_{k}^{n}$. This process is continued and we obtain a continuum $X$ with the required properties. It follows from the proof of Theorem 1 in [5] that $X$ has property $Q$ (weak) and hence has CIP. We now show that the cone over $X$, denoted by Cone $(X)$, does not have CIP.

By Cone $(X)$ we mean the identification space obtained by taking the disjoint union of $X \times I$ and a set consisting of a single point $v$, and then identifying each point of the form $(x, 1)$ with the point $v$. The point $v$ is called the vertex of Cone $(X)$ and, if $0 \leqq t<1$, we shall regard $X \times[0, t]$ as a subspace of Cone $(X)$. Moreover, since $X$ is a compact metric space, we may assume that Cone $(X)$ is embedded in $E^{\omega}$.

Suppose Cone $(X)$ has CIP and $f:$ Cone $(X) \rightarrow$ Cone $(X)$ is a mapping whose fixed point set is $X \times\{0\}$. Since the fixed point set of $f$ is $X \times\{0\}$, it follows that there is a number $q$ such that $0<q<1$ and $f^{-1}(v) \subset Y$ where

$$
Y=\{(x, t) \mid(x, t) \in \text { Cone }(X) \text { and } t \geqq q\} .
$$

We note that for a point of the form $(x, q), f(x, q)=(x, s)$ for some $s>q$. Let $\alpha$ : Cone $(X) \rightarrow Y$ be a retraction of Cone $(X)$ onto $Y$ defined by

$$
\alpha(x, t)=\left\{\begin{array}{l}
(x, t) \text { if } q \leqq t \leqq 1 \\
(x, q) \text { i } \text { f } 0 \leqq t \leqq q
\end{array}\right.
$$

Let $g: Y \rightarrow Y$ be the mapping defined by $g(x, t)=\alpha f(x, t)$ for all $(x, t) \in Y$. It follows that $g$ is a fixed point free map and, consequently, there is a number $\varepsilon>0$ such that $\rho(g(x, t),(x, t)) \geqq \varepsilon$ for every point $(x, t) \in Y$. From the construction of $X$ it follows that there is a polyhedron $P \subset X$ and a retraction $r_{1}: X \rightarrow P$ from $X$ onto $P$ such that $\rho\left(r_{1}(x), x\right)<\varepsilon / 2$ for all $x \in X$. Define a mapping $r_{2}: Y \rightarrow$ $Y$ by $r_{2}(x, t)=\left(r_{1}(x), t\right)$ for all $(x, t) \in Y$. Then $K=r_{2}(Y)$ is an $A R$ space which is homeomorphic to Cone $(P)$. Let $h: K \rightarrow K$ be the map defined by $h(x, t)=r_{2} g(x, t)$ for all $(x, t) \in K$. Then $\rho(h(x, t)$, $(x, t))>\varepsilon / 2$ for all $(x, t) \in K$. But this contradicts the fact that $K$ has the fixed point property and, therefore, Cone $(X)$ does not have 
CIP.

The following examples are higher dimensional analogues of Example 4.1.

EXAMPLE 4.2. If we follow the construction used in Example 4.1 and replace $A^{1}$ by $A^{n}$, then we obtain an $n$-dimensional $L C^{n-1}$ continuum $X^{n}$ such that Cone $\left(X^{n}\right)$ does not have CIP. In a similar fashion, one can replace $A^{1}$ by $A_{1}^{\infty}, B_{k}^{n}$ by a copy of $A_{n+k}^{\infty}$, and modify the construction of Example 4.1 to obtain an $L C^{\infty}$ continuum $X^{\infty}$ such that Cone $\left(X^{\infty}\right)$ does not have CIP. A modification of the proof of Theorem 1 in [5] as applied to Example 4.1, together with the ideas introduced in 3.6, can be used to prove that $X^{n}$ and $X^{\infty}$ have property $Q$ (weak) and hence have CIP. We omit the details.

5. Deformation retracts. In $[4, p .1024]$ it is shown that CIP is not preserved by strong deformation retractions of non-locally connected continua. The following example shows that for each positive integer $n=1,2,3, \cdots$, there is a contractible $(n+2)$-dimensional $L C^{n-1}$ continuum $Z^{n}$ having CIP which contains a strong deformation retract not having CIP.

EXAMPLE 5.1. Let $X^{n}$ denote the $n$-dimensional $L C^{n-1}$ continuum defined in 4.2 and let $B^{n}$ denote the $(n+1)$-dimensional $L C^{n-1}$ continuum defined by R. J. Knill in [2, p. 37]. Define $Z^{n}$ to be the wedge obtained by taking the disjoint union of Cone $\left(X^{n}\right)$ and Cone $\left(B^{n}\right)$, and then identifying the vertex of Cone $\left(X^{n}\right)$ with the vertex of Cone $\left(B^{n}\right)$. Clearly, $Z^{n}$ is a contractible $(n+2)$-dimensional $L C^{n-1}$ continuum containing Cone $\left(X^{n}\right)$ as a strong deformation retract. Since Cone $\left(X^{n}\right)$ does not have CIP, to complete the proof we must show that $Z^{n}$ has CIP. But, since Cone $\left(B^{n}\right)$ is an arcwise connected continuum without the fixed point property [2, p. 40], it follows from Proposition 3.6 of $[4, p .1022]$ that $Z^{n}$ has CIP if Cone $\left(B^{n}\right)$ has CIP. We now show that Cone $\left(B^{n}\right)$ has CIP.

Let $v$ denote the vertex of Cone $\left(B^{n}\right)$ and let $K$ be a nonempty closed subset of Cone $\left(B^{n}\right)$. If $v \in K$, then $K$ is a fixed point set of Cone $\left(B^{n}\right)$ by Theorem 1 of $[8, p$. 554]. Thus, without loss of generality, we shall assume that $K \subset B^{n} \times[0,1 / 4]$. It is easy to show, using Lemma 2.1 of [4, p. 1018], that there is a mapping $f_{0}: B^{n} \times$ $[0,1 / 2] \rightarrow$ Cone $\left(B^{n}\right)$ such that the fixed point set of $f_{0}$ is $K$ and $f_{0}\left(B^{n} \times\{1 / 2\}\right)=v$. For $0<t \leqq 1 / 4$, let

$$
C_{t}^{n}=\left\{(x, s) \mid(x, s) \in \text { Cone }\left(B^{n}\right) \text { and } s \geqq 1-t\right\}
$$

and let $h_{t}$ : Cone $\left(B^{n}\right) \rightarrow C_{t}^{n}$ be the homeomorphism defined by 


$$
h_{t}(x, s)=(x, t s+1-t) \text { for all }(x, s) \in \text { Cone }\left(B^{n}\right) .
$$

Let $g^{n}$ : Cone $\left(B^{n}\right) \rightarrow$ Cone $\left(B^{n}\right)$ denote the fixed point free map defined by R. J. Knill in [2, p. 40]. Then $f_{t}=h_{t} g^{n} h_{t}^{-1}$ is a fixed point free self-map of $C_{t}^{n}$. Let $\sigma(t)=t-1 / 2$ if $1 / 2<t<3 / 4$, and $\sigma(t)=1 / 4$ if $3 / 4 \leqq t \leqq 1$. Define a function $f:$ Cone $\left(B^{n}\right) \rightarrow$ Cone $\left(B^{n}\right)$ by

$$
f(x, t)= \begin{cases}f_{0}(x, t) & \text { if } 0 \leqq t \leqq \frac{1}{2}, \\ f_{\sigma(t)}\left(x, \frac{3}{2}-t\right) & \text { if } \frac{1}{2}<t \leqq \frac{3}{4} \\ f_{\sigma(t)}(x, t) & \text { if } \frac{3}{4} \leqq t \leqq 1\end{cases}
$$

It follows that $f$ is continuous and that the fixed point set of $f$ is $K$.

REMARKS 6 . We end the paper with the following three remarks.

6.1. By using only spheres of dimension $\geqq n+1$ in the constructions found in 3.6 and 4.2, it is possible to construct examples which are both $C^{n}$ and $L C^{\infty}$.

6.2. The authors do not know of any locally contractible examples.

6.3. The question posed in [3, p. 165] as to whether every $A R$-space ( $A N R$-space) has CIP remains unsolved.

\section{REFERENCES}

1. K. Borsuk, Theory of retracts, Monografie Matematyczne 44, Warszawa 1967.

2. R. J. Knill, Cones, products and fixed points, Fund. Math., 60 (1967), 35-46.

3. J. R. Martin, Fixed point sets of Peano continua, Pacific J. Math., 74 (1978), 163-166.

4. J. R. Martin and S. B. Nadler, Jr., Examples and questions in the theory of fixed point sets, Canad. J. Math., 31 (1979), 1017-1032.

5. J. R. Martin and E. D. Tymchatyn, Fixed point sets of 1-dimensional Peano continua, Pacific J. Math., 89 (1980), 147-149.

6. H. Schirmer, Fixed point sets of homeomorphisms of compact surfaces, Israel J. Math., 10 (1971), 373-378.

7. - Fixed point sets of polyhedra, Pacific J. Math., 52 (1974), 221-226.

8. L. E. Ward, Jr., Fixed point sets, Pacific J. Math., 47 (1973), 553-565.

Received May 15, 1981. The first author was partially supported by NSERC Grant A 8205 , and the second and third authors were partially supported by NSERC Grant A5616.

UNIVERSITY OF SASKatchewaN

Saskatoon, Saskatchewan

CANAda S7N 0W0 



\section{PACIFIC JOURNAL OF MATHEMATICS}

\section{EDITORS}

DONALD BABBITT (Managing Editor)

J. DUGUNDJI

University of California

Los Angeles, California 90024

Hugo Rossi

University of Utah

Salt Lake City, UT 84112

C. C. Moore and Arthur Agus

Department of Mathematics

University of Southern California

Los Angeles, California 90007

R. FinN and J. Milgram

Stanford University

Stanford, California 94305

University of California

Berkeley, CA 94720

\section{ASSOCIATE EDITORS}
R. ARNES
E. F. BeCKenBaCH
B. H. NEUMANN
F. WoLF
K. YoshidA

\section{SUPPORTING INSTITUTIONS}

UNIVERSITY OF ARIZONA

UNIVERSITY OF BRITISH COLUMBIA

CALIFORNIA INSTITUTE OF TECHNOLOGY

UNIVERSITY OF CALIFORNIA

MONTANA STATE UNIVERSITY

UNIVERSITY OF NEVADA, RENO

NEW MEXICO STATE UNIVERSITY

OREGON STATE UNIVERSITY
UNIVERSITY OF OREGON

UNIVERSITY OF SOUTHERN CALIFORNIA

STANFORD UNIVERSITY

UNIVERSITY OF HAWAII

UNIVERSITY OF TOKYO

UNIVERSITY OF UTAH

WASHINGTON STATE UNIVERSITY

UNIVERSITY OF WASHINGTON 


\section{Pacific Journal of Mathematics}

\section{Vol. 101, No. $1 \quad$ November, 1982}

Natália Bebiano, On the evaluation of permanents $\ldots \ldots \ldots \ldots \ldots \ldots \ldots \ldots$

David Borwein and Bruce Brigham Watson, Tauberian theorems between

the logarithmic and Abel-type summability methods $\ldots \ldots \ldots \ldots \ldots \ldots 11$

Leo George Chouinard, II, Hermite semigroup rings $\ldots \ldots \ldots \ldots \ldots \ldots \ldots$

Kun-Jen Chung, Remarks on nonlinear contractions $\ldots \ldots \ldots \ldots \ldots \ldots . \ldots 4$

Lawrence Jay Corwin, Representations of division algebras over local

fields. II ......................................... 49

Mahlon M. Day, Left thick to left lumpy—a guided tour $\ldots \ldots \ldots \ldots \ldots 71$

M. Edelstein and Mo Tak Kiang, On ultimately nonexpansive

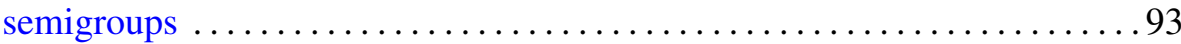

Mary Rodriguez Embry, Semigroups of quasinormal operators . ........ 103

William Goldman and Morris William Hirsch, Polynomial forms on

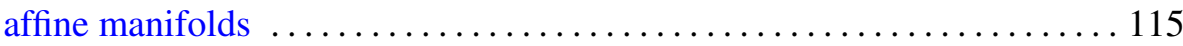

S. Janakiraman and T. Soundararajan, Totally bounded group topologies

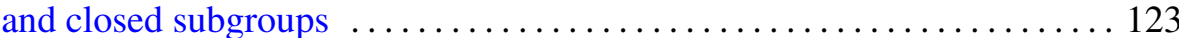

John Rowlay Martin, Lex Gerard Oversteegen and Edward D.

Tymchatyn, Fixed point set of products and cones $\ldots \ldots \ldots \ldots \ldots \ldots 133$

Jan van Mill, A homogeneous Eberlein compact space which is not metrizable ........................................ 141

Steven Paul Plotnick, Embedding homology 3-spheres in $S^{5} \ldots \ldots \ldots \ldots 147$

Norbert Riedel, Classification of the $C^{*}$-algebras associated with minimal rotations

Benedict Seifert, Combinatorial and geometric properties of weight systems of irreducible finite-dimensional representations of simple split Lie algebras over fields of 0 characteristic

James E. Simpson, Dilations on locally convex spaces

Paolo M. Soardi, Schauder bases and fixed points of nonexpansive mappings

Yoshio Tanaka, Point-countable $k$-systems and products of $k$-spaces

Fausto A. Toranzos, The points of local nonconvexity of starshaped sets . . . 209

Lorenzo Traldi, The determinantal ideals of link modules. I . . . . . . . . 215

P. C. Trombi, Invariant harmonic analysis on split rank one groups with applications

Shinji Yamashita, Nonnormal Blaschke quotients 